\title{
Mixed ab initio and semiempirical study of hydrogen-terminated finite germanium nanowires
}

\author{
Shanawer Niaz ${ }^{1,2}$,a , Oğuz Gülseren ${ }^{2}$, Muhammad Aslam Khan ${ }^{3}$, and Irfan Ullah ${ }^{4}$ \\ 1 Department of Physics, University of Sargodha, Sub-campus Bhakkar 30000, Pakistan \\ 2 Department of Physics, Bilkent University, Ankara 06800, Turkey \\ 3 Department of Physics, Khawaja Fareed University of Engineering and Information Technology, Abu Dhabi Road, Rahim \\ Yar Khan, 64200, Pakistan \\ ${ }^{4}$ Department of Chemistry, University of Sargodha, Sub-campus Bhakkar 30000, Pakistan
}

Received: 5 March 2018 / Revised: 8 August 2018

Published online: 2 November 2018

(C) Società Italiana di Fisica / Springer-Verlag GmbH Germany, part of Springer Nature, 2018

\begin{abstract}
We present a mixed $a b$ initio and semiempirical method for the cohesive energy and electronic gap calculations of hydrogen passivated tetrahedral and clathrate germanium nanowires ( $\sim 1850$ atoms $)$ with acceptable accuracy, comparable to density functional theory results, and with a significantly lower computational cost. First, we find that the PM6 semiempirical method produce the most accurate geometries when compared with the DFT results; whereas other semiempirical methods such as AM1, PM3 and PM7 clearly underestimate (or overestimate). Second, we implement the DFT@PM6 mixed scheme for cohesive/binding energy and electronic gap calculations which shows promising results compared with reference values of DFT. However, the bulk energy gap and binding energy values from the quantum confinement fitting procedure slightly underestimate the results which can be easily overcome using suitable functional and basis set/ECP. Also, a comparison with previous work clearly shows that the calculated electronic gap for bulk germanium is extremely sensitive to the choice of framework. Further development in this research work is progressing.
\end{abstract}

\section{Introduction}

Silicon nanowires (SiNWs) are particularly attractive for nanoelectronics because their diameter and electronic properties can be controlled during synthesis in a predictable manner. This ability has been employed for the sensors, logic gates and field-effect transistors (FETs) [1-3]. Like silicon, germanium is also a Group 14 semiconductor material and as such, it shares quite a lot of properties in common with silicon, such as a diamond like cubic crystal structure. Germanium also exhibits several properties that are superior to those of silicon, including higher charge carrier mobility. Because of this reason, interest in germanium nanowires has thrived in among research groups and industry from the last few years to advance functionality in electronic devices. Hence, germanium nanowires (GeNWs) have already shown potential applications due to their higher electron-hole mobility and larger exciton Bohr radius [4-6]. GeNWs have numerous potential applications based on the electrochemical, electrical, and magnetic properties such as spintronics [7], solar cells with enhanced efficiency [8], Li-ion batteries [9], photodetectors in the visible range [10], flexible chemical sensors [11].

Germanium nanowires are used in terms of studying strain and dopant location, including transport modulation and band offset efficiency in the form of core/shell nanowires [12]. Due to the high surface to volume ratio, surface chemistry can much affect nanowire properties, therefore research into the functionalization of germanium nanowire surfaces has resulted in further development and a better understanding of the potential of these materials [13]. To implement GeNWs in electronics applications, doping is one of the processes to increase its conductivity and efficiency of devices. Several research groups fabricated the high-performance GeNW field effect transistors (FETs) with both n- and p-doped GeNWs $[14,15]$. However, as the nanowire diameter decreases, the doping process becomes harder and less reliable. One of the alternative ways to control the electronic properties and avoid doping process is by

\footnotetext{
a e-mail: shanawersi@gmail.com (corresponding author)
} 
applying strain to the germanium nanowire [16]. Chemical passivation is another efficient way of tuning the electronic properties of GeNWs. In this approach, only the nanowire surface is modified while the core of the nanowire remains intact as compared to the doping technique where both the surface and the core are considered. For GeNWs, surface modifications with hydrogen, halogen and organic chemicals have been studied in experiments and theories [17-27].

Despite such clear device potential, as is mentioned above, relatively a few ab initio calculations of optical properties beyond the one-particle approach have been performed so far to clarify the experimental evidences and investigate the potential applications of such nanoscale materials. In fact, the theoretical scheme is essentially based either on $a b$ initio calculations, which neglect the electron-hole Coulomb interaction effects (which instead is expected to play an important role due to the reduced dimensionality of such systems) or within effective mass approximation (EMA) calculations and semi-empirical approaches. The theoretical design of more complex systems, involving several nanoscale components for well-specified operations, using all-electron $a b$ initio or density functional theory (DFT) calculations has a prohibitively high computational cost. Compared to silicon $(Z=14)$ this problem is more acute for germanium $(Z=32)$ because of the larger number of electrons, which make Ge-nanowires calculations much more computationally demanding for DFT calculations, even with minimal basis sets. We have put considerable effort to overcome this difficulty, by the implementation of suitable effective core potentials and application of appropriate (semiempirical) model chemistry, with no (significant) compromise on accuracy and quality of results. To this end, we have tested a large variety of atomic core potentials in conjunction with available basis sets, and we have evaluated the performance of several semiempirical methods, before our final choice of adopting a "mixed" scheme of DFT and semiempirical calculations. In this paper, we present detailed results for the structural, cohesive and electronic properties of germanium nanowires. We have considered these properties of representative tetrahedral or "bulk-like" and some clathrate-type or "fullerene-like" germanium nanowires. Clathrates are class of low density "cage-like" allotropes of Ge, which have direct or nearly direct band gaps and thus the possibility for strong optical absorption and emission. Therefore, we especially focus on size-dependent cohesive and electronic properties of H-GeNWs using mixed ab initio and semiempirical techniques.

\section{Theoretical and computational methods}

We have performed structural optimizations in the framework of semiempirical models whereas single point energies of which were calculated using the density functional theory (DFT), i.e. DFT-PBE@PM6 for ultra-thin hydrogen passivated germanium nanowires $(\mathrm{H}-\mathrm{GeNWs})$ of finite lengths grown along the $\left[\begin{array}{lll}1 & 1 & 0\end{array}\right]$ bulk direction (including some selected cases of clathrate H-GeNWs). The results from the DFT calculations were used as a reference to facilitate the comparison of the performance of the semiempirical methods on the systems under study.

For the DFT computations the gradient corrected functional of Perdew, Burke and Ernzerhof (PBE) [28] was employed using the Stuttgart-Dresden (SDD) basis set [29,30], which describes the inner 28 core electrons with a pseudopotential. Tight convergence criteria were placed for the maximum norm of the Cartesian gradient (up to $10^{-4}$ a.u.). Fine convergence criteria were used for the SCF energy (up to $10^{-7}$ a.u.) as well as the ones for electron density (rms of the density matrix less than $10^{-6}$ ). All DFT calculations were performed with the GAUSSIAN 09 program package [31]. The primary semiempirical method employed is Austin Model 1 (AM1) [32,33], while for the comprehensive comparison we also included Parameterization Method 3 (PM3) [34], Parameterization Method 6 (PM6) [35] and Parameterization Method 7 (PM7) [36]. The convergence criterion placed on the Cartesian gradient for the semiempirical calculations was $0.01 \mathrm{kcal} / \mathrm{mol} / \AA\left(8.4 \times 10^{-6}\right.$ a.u. $)$ for Ge. All the semiempirical calculations were performed using MOPAC 2012 [37] and MOPAC 2016 [38]. Partial density of states (PDOS) calculations were performed using Multiwfn v3.3.9 [39] and reproduced using GaussSum v2.2 [40].

\section{Results and discussions}

We have studied various properties of hydrogen passivated germanium nanowires such as structural, electronic and cohesive properties. Nanowires have been categorized in terms of their size, i.e. diameter and length (aspect ratio) of nanowires. For example, diameter varies between the range of $0.99 \mathrm{~nm}$ and $2.18 \mathrm{~nm}$ whereas each nanowire has three different length ranges between $4.23 \mathrm{~nm}$ and $8.20 \mathrm{~nm}$ (cross-sectional view of geometries can be seen in fig. 1).

\subsection{Model selection and structural properties}

We have constructed hydrogen-terminated, finite, ultrathin, germanium (tetrahedral and clathrate) nanowires of various sizes. The growth direction for this work is $\left[\begin{array}{lll}1 & 1 & 0\end{array}\right]$ of the bulk germanium. All of the NWs examined here have a closed-structure cross-section with both perpendicular surface germanium-dihydride groups (tetrahedral) and germanium monohydride group (clathrates). As far as clathrate-type germanium nanowires are concerned, in this study, we consider type-I clathrate which is a simple cubic lattice with 46 atoms per unit cell and the $\operatorname{Pr} \overline{3} n$ (No. 223) group symmetry. 


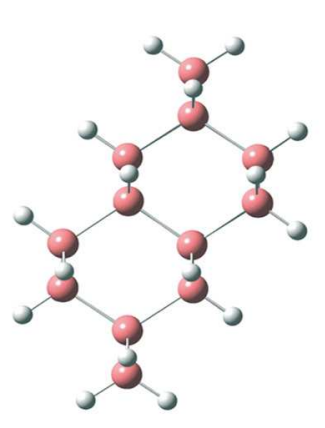

(a)

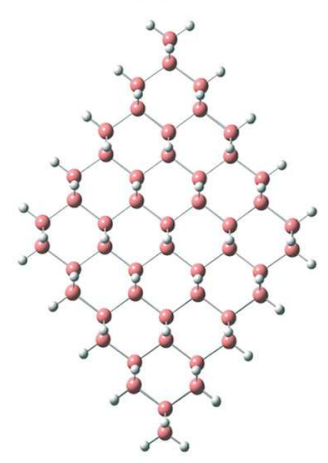

(d)

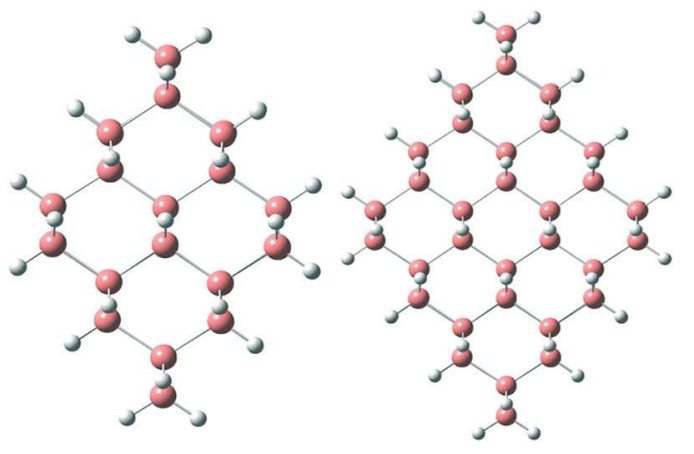

(c)

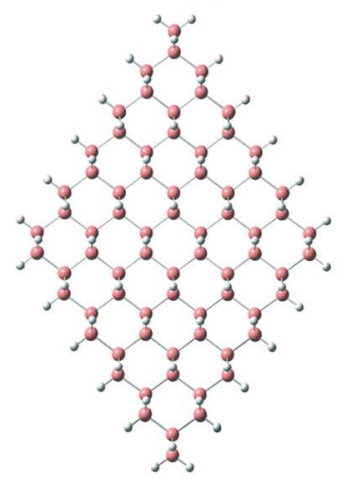

(e)

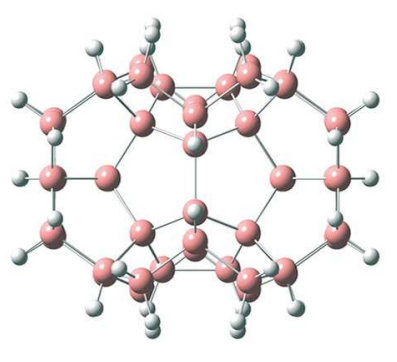

(f)

Fig. 1. Cross-sectional view of fully optimised structures of hydrogen passivated finite germanium nanowires (a)-(e) tetrahedral and (f) clathrate. The approximate diameters, D, are $0.99 \mathrm{~nm}-2.7 \mathrm{~nm}$ whereas every respective diameter has three length ranges, i.e. $\mathrm{L} 10=4.23 \mathrm{~nm}, \mathrm{~L} 15=6.22 \mathrm{~nm}$ and $\mathrm{L} 21=8.20 \mathrm{~nm}$.

In figs. 1(a)-(e) represent tetrahedral whereas (f) corresponds to the clathrate type H-GeNWs. The diameters are (a) $0.99 \mathrm{~nm}$ (b) $1.24 \mathrm{~nm}$ (c) $1.71 \mathrm{~nm}$ (d) $2.18 \mathrm{~nm}$ (e) $2.64 \mathrm{~nm}$ and (f) $1.20 \mathrm{~nm}$, respectively. The number of atoms ranges in terms of Ge:H ratio for lengths L10, L15, L21 are (a) 122:110, 182:160, 242:210 (b) 187:140, 277:200, 385:272 (c) $334: 196,494: 276,718: 388$ (d) 523:256, 773:356, 973:436, (e) 754:320, 1114:440, 1330:512 and (f) 190:136, 282:192, 374:248, whereas lengths are L10 $=4.23 \mathrm{~nm}, \mathrm{~L} 15=6.22 \mathrm{~nm}$, and L21 $=8.20 \mathrm{~nm}$. In the ball and stick model, the pink balls are Ge and the white ones are hydrogen atoms. There is a significant difference between geometries of tetrahedral and clathrate GeNWs which is the surface of nanowires, i.e. the first ones have a mixture of dihydride and monohydrides whereas the second ones contain monohydrides only. Furthermore panels (a)-(e) have $\mathrm{C}_{2 \mathrm{v}}$ symmetry whereas (f) carry $D_{2 h}$ symmetry group. In both cases, the surface of every nanowire is completely covered with passivants (i.e., hydrogen atoms) so that no dangling bond or bare surface germanium atom is remaining.

The structural differences between the optimized geometries of each method can be explored by plotting germaniumgermanium bond length and bond angle distribution diagrams. Figure 2 shows the Ge-Ge bond length distributions for both (a) tetrahedral- and (b) clathrate-type NWs, respectively, which are optimized using the AM1, PM3, PM6, PM7 and DFT/PBE methods. The AM1 and PM3 methods completely failed to keep the symmetry of the initial structure and gave wrong geometry whereas PM7 somewhat succeeded with wrong interpretation of bond length (and bond angle) distribution! Hence there is no need to even mention the bond lengths for these three methods. From now on we will discuss PM6 and DFT calculations only, so the average Ge-Ge bond length of the PM6 structures is $2.44 \AA$ ( $2.46 \AA$ for the clathrate type), whereas the DFT/PBE structure is $2.47 \AA$ ( $2.52 \AA$ for the clathrate type). This is clear from the values and eye contact with the plots that PM6 follows DFT (and in agreement with slight deviations) structures also comparable with the experimental value of Ge-Ge bond length which is $2.45 \AA$. In general, PM6 gives the remarkably good average Ge-Ge bond length with a standard deviation of $\sigma_{\mathrm{PM} 6}=0.01 \AA$ (which seems promising), however, with a broader distribution of values compared with the reference $\mathrm{DFT} / \mathrm{PBE}$ geometry, i.e. $\sigma_{\mathrm{PBE}}=0.02 \AA$.

The corresponding bond angle distributions are shown in fig. 3(a) for tetrahedral and (b) for clathrate Ge-NWs, respectively, which are optimized using AM1, PM3, PM6, PM7 and DFT/PBE methods. The average Ge-Ge bond angle of PM6 structures is $108.9^{\circ}\left(107.2^{\circ}\right.$ for clathrate type) and for the case of DFT/PBE structure it is $109.6^{\circ}$ $\left(107.5^{\circ}\right.$ for clathrate type), whereas the experiment Ge-Ge bond angle value of the tetrahedral structure is $109.5^{\circ}$. We can interpret that the PM6 method is in agreement with the DFT level calculations and these values are also comparable to the bulk Ge-Ge bond angle value with a standard deviation of $\sigma_{\mathrm{PM} 6}=0.06$ degrees. 

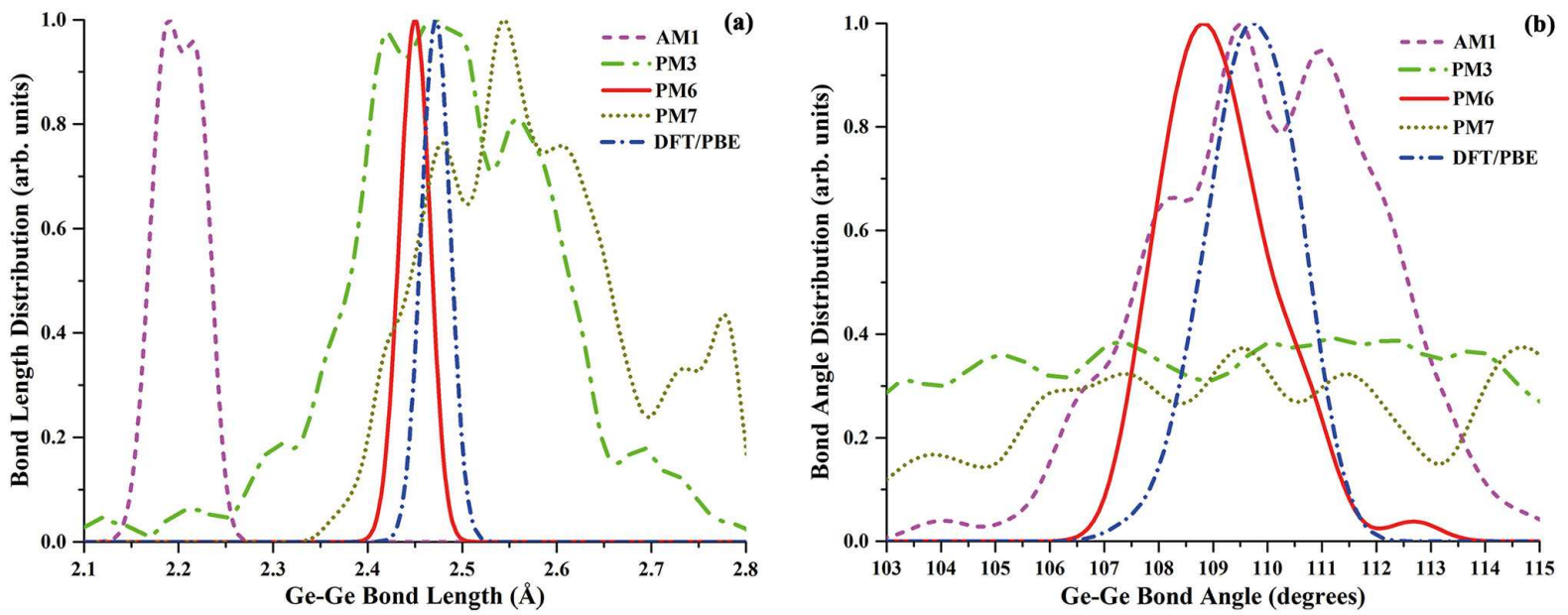

Fig. 2. Ge-Ge bond length (a) and bond angle (b) distribution of tetrahedral hydrogen passivate germanium nanowire.
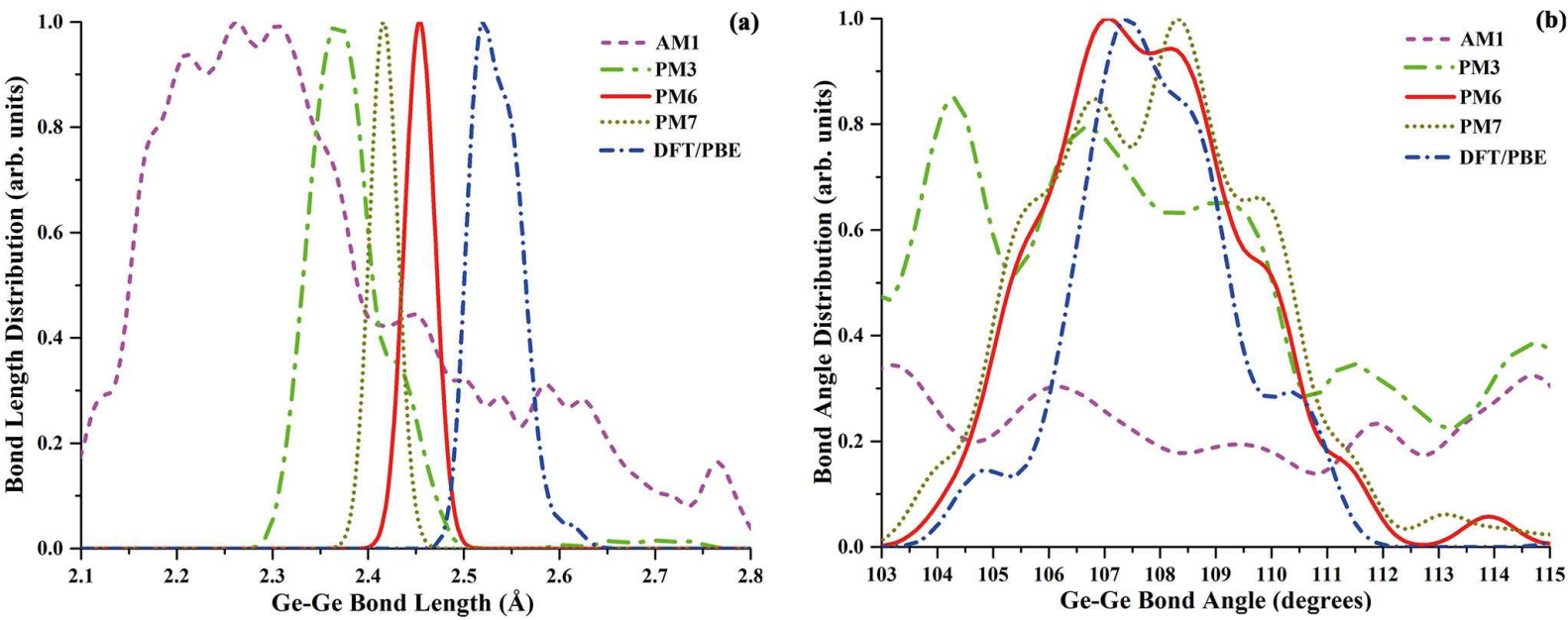

Fig. 3. Ge-Ge bond length (a) and bond angle (b) distribution of clathrate hydrogen passivate germanium nanowire.

Table 1. Comparison (as a test case) of the total energies and HOMO-LUMO gaps of very small tetrahedral H-terminated finite germanium nanowires using DFT@PM6, PM6 with DFT methods.

\begin{tabular}{|c|c|c|c|c|c|c|c|c|c|}
\hline \multirow{2}{*}{ Type } & \multirow{2}{*}{ Sym. } & \multicolumn{2}{|c|}{$\mathrm{DFT}^{(\mathrm{a})}$} & \multicolumn{2}{|c|}{$\mathrm{DFT}^{(\mathrm{a})} @ \mathrm{PM} 6$} & \multicolumn{2}{|c|}{$\begin{array}{l}\text { Difference }\left(\mathrm{DFT}^{(\mathrm{a})} \text { - }\right. \\
\left.\mathrm{DFT}^{(\mathrm{a})} \mathrm{PM} 6\right) / \text { atom }\end{array}$} & \multicolumn{2}{|c|}{ PM6 } \\
\hline & & $\begin{array}{c}\text { Total energy } \\
(\mathrm{eV})\end{array}$ & $\begin{array}{c}\text { H-L gap } \\
(\mathrm{eV})\end{array}$ & $\begin{array}{c}\text { Total energy } \\
(\mathrm{eV})\end{array}$ & $\begin{array}{c}\text { H-L gap } \\
(\mathrm{eV})\end{array}$ & $\begin{array}{c}\text { Total energy } \\
\text { (eV/atom) }\end{array}$ & $\begin{array}{c}\text { H-L gap } \\
(\mathrm{eV} / \text { atom })\end{array}$ & $\begin{array}{c}\text { Total energy } \\
\qquad(\mathrm{eV})\end{array}$ & $\begin{array}{c}\text { H-L gap } \\
(\mathrm{eV})\end{array}$ \\
\hline & (a) $\mathrm{C}_{2 \mathrm{~h}}$ & -2374148.25 & 4.16 & -2374147.35 & 4.28 & 0.021 & 0.003 & -3995.40 & 6.89 \\
\hline & (b) $\mathrm{C}_{2 \mathrm{v}}$ & -4465367.11 & 3.63 & -4465365.37 & 3.68 & 0.022 & 0.0006 & -7269.77 & 6.51 \\
\hline
\end{tabular}

(a) $\mathrm{DFT} / \mathrm{PBE} / \mathrm{SDD}$.

It is worth mentioning that, technically, the bond length and bond angle value for the tetrahedral type structure may differ. Taking into account rather accurate results of PM6 on the specific systems, as well as the significant lower computation demands of the method, we can conclude that the method of choice is PM6 (for geometry optimizations) that would allow acceptable accurate calculations with realistic computational timings needed for larger systems. Systematic comparison (as a test case) of the total energies and HOMO-LUMO gaps of very small tetrahedral Hterminated finite germanium nanowires using DFT@PM6, PM6 with DFT is represented in table 1. The results, in general, clearly suggest that DFT-PBE@PM6 is the method of choice to calculate energies and electronic gaps for larger NWs in terms of both computational cost and accuracy. 


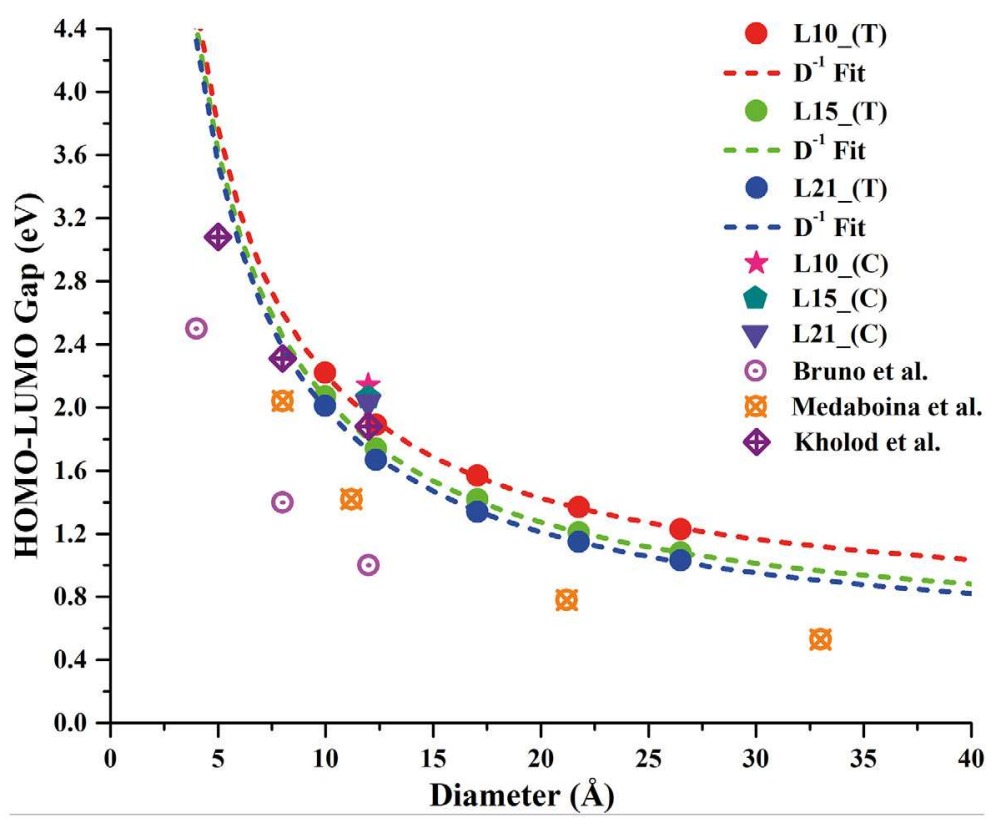

Fig. 4. Shows HOMO-LUMO gap dependence on the diameter of all three different lengths of H-GeNWs. The red, green and blue dots represent tetrahedral $(\mathrm{T})$ whereas the pink star, cyan pentagon and violet triangle represent clathrate (C) type nanowires, respectively. The dotted lines in (a) show $D^{-1}$ linear dependence fitted to our results using eq. (1). Furthermore, magenta circle, orange crossed circle and purple crossed square represent results from Bruno et al. [45], Medaboina et al. [46] and Kholod et al. [47] for comparison, respectively. The DFT-PBE@PM6 method is used for calculations.

\subsection{Electronic properties}

Figure 4 shows the size dependence of electronic gaps (HOMO-LUMO gap) with respect to their diameter for tetrahedral (T) and some random points for clathrate $(\mathrm{C})$ type hydrogen passivated germanium nanowires. Clearly from the figure, the HOMO-LUMO gap is decreasing as far as the diameter of GeNWs is increasing. In comparison, the electronic gap of clathrate H-GeNWs is higher than the tetrahedral H-GeNWs of the same sizes, see table 2. These results follow the quantum confinement (QC) effect theory. As was discussed in our previous work [41,42] using quantum confinement concept, the extrapolation formula of our ab initio results can be described by the expected dependence of the HOMO-LUMO gap on size (number of atoms and/or diameter) as

$$
\begin{aligned}
& E(D)_{N W}=C+\left(E \times D^{-1}\right), \quad \text { or } \\
& E(N)_{N W}=B+\left(F \times N^{-\frac{1}{3}}\right),
\end{aligned}
$$

where $D$ is the diameter, $N$ is the total number of Ge atoms, $E(D)_{N W}$ is the predicted HOMO-LUMO gap energy and $E(N)_{N W}$ is the predicted binding (or cohesive) energy of the hydrogen passivated germanium nanowire whereas $C, B, E$ and $F$ are used as adjustable parameters to be determined by the fit.

As we can see in eqs. (2)-(4) the HL gap (parameter $C$ ) of tetrahedral H-GeNWs is reducing from $0.66 \mathrm{eV}$ to $0.43 \mathrm{eV}$ as far as the length of nanowire is increasing which is indeed less than the experiment bandgap value which is $0.74 \mathrm{eV}$ (at $0 \mathrm{~K}$ ) and $0.66 \mathrm{eV}$ (at $300 \mathrm{~K}$ ) [43]. In our previous research work, the HOMO-LUMO gap and optical gap of hydrogen passivated GeQDs [44] and even HOMO-LUMO gap of small H-GeNWs [17] is higher than the current gap values within the same diameter considerations. As was reported earlier [41], from eq. (1) we can have accurate enough speculations of energy gap values (and/or cohesive energy) of bulk crystal by extrapolating small points. But it is necessarily important to select the appropriate model for electronic structure calculations for such an electron rich element like germanium. It is revealed from our current results that the basis set/effective core potential (ECP) along with the PBE functional underestimated electronic gap (and binding/cohesive energies). In the light of our previous experience $[17,41,42,44]$, calculations may be performed using hybrid functional such as B3LYP along with good quality basis set, for example, Split Valence Polarization (SVP) or Triple-Zeta Valence Polarization (TZVP), etc., or even using better ECP, which is, unfortunately, not available and still in development phase. Due to the reasons mentioned in the last paragraphs of the introduction section, we may consider expensive models in further studies therefore the work is in progress. Secondly, we have just considered only few diameters (five in this case), but probably more diameters are to considered, in order to improve the accuracy. 
Table 2. Structural (total number of atoms (i.e. germanium + hydrogen atoms), symmetry, diameter, length, aspect ratio), energetic (cohesive energy per germanium atom, binding energy per germanium atom) and electronic (HOMO-LUMO gap) characteristics of tetrahedral and clathrate type H-terminated finite germanium nanowires. The DFT-PBE@PM6 method is used for calculations.

\begin{tabular}{|c|c|c|c|c|c|c|c|c|c|}
\hline \multicolumn{2}{|c|}{ Nanowires } & Sym. & $\begin{array}{l}\text { Length } \\
(\AA)\end{array}$ & $\begin{array}{c}\text { Diameter } \\
(\AA)\end{array}$ & $\begin{array}{c}\text { Aspect ratio } \\
\text { (L:D) }\end{array}$ & $\begin{array}{c}\text { Total number } \\
\text { of atoms }\end{array}$ & \begin{tabular}{|c|} 
Binding energy \\
$(\mathrm{eV} / \mathrm{Ge})$
\end{tabular} & $\begin{array}{c}\text { Cohesive energy } \\
(\mathrm{eV} / \mathrm{Ge})\end{array}$ & $\begin{array}{c}\text { HOMO-LUMO gap } \\
(\mathrm{eV})\end{array}$ \\
\hline \multirow{15}{*}{ Tetrahedral } & \multirow{5}{*}{ L10_(T) } & \multirow{5}{*}{$\mathrm{C}_{2 \mathrm{v}}$} & \multirow{5}{*}{42.29} & \begin{tabular}{|l|}
9.98 \\
\end{tabular} & 4.22 & 232 & 5.24 & 2.42 & 2.22 \\
\hline & & & & 12.35 & 3.42 & 327 & 4.92 & 2.58 & 1.89 \\
\hline & & & & 17.05 & 2.48 & 530 & 4.59 & 2.75 & 1.57 \\
\hline & & & & 21.77 & 1.95 & 779 & 4.39 & 2.86 & 1.37 \\
\hline & & & & 26.49 & 1.60 & 1074 & 4.25 & 2.93 & 1.23 \\
\hline & \multirow{5}{*}{ L15_(T) } & \multirow{5}{*}{$\mathrm{C}_{2 \mathrm{v}}$} & \multirow{5}{*}{62.16} & 9.98 & 6.21 & 342 & 5.19 & 2.44 & 2.07 \\
\hline & & & & 12.35 & 5.03 & 477 & 4.87 & 2.61 & 1.74 \\
\hline & & & & 17.05 & 3.65 & 770 & 4.53 & 2.78 & 1.42 \\
\hline & & & & 21.77 & 2.86 & 1129 & 4.33 & 2.89 & 1.21 \\
\hline & & & & 26.49 & 2.35 & 1554 & 4.19 & 2.96 & 1.08 \\
\hline & \multirow{5}{*}{ L21_(T) } & \multirow{5}{*}{$\mathrm{C}_{2 \mathrm{v}}$} & \multirow{5}{*}{82.02} & 9.98 & 8.20 & 452 & 5.17 & 2.45 & 2.01 \\
\hline & & & & 12.35 & 6.96 & 657 & 4.83 & 2.62 & 1.67 \\
\hline & & & & 17.05 & 5.28 & 1106 & 4.49 & 2.80 & 1.34 \\
\hline & & & & 21.77 & 3.59 & 1409 & 4.30 & 2.90 & 1.15 \\
\hline & & & & 26.49 & 2.80 & 1842 & 4.17 & 2.97 & 1.03 \\
\hline \multirow{3}{*}{ Clathrate } & L10_(C) & $\mathrm{D}_{2 \mathrm{~h}}$ & 42.29 & 11.98 & 3.76 & 326 & 4.84 & 2.82 & 2.14 \\
\hline & L15_(C) & $\mathrm{D}_{2 \mathrm{~h}}$ & 62.16 & 11.98 & 5.53 & 474 & 4.77 & 2.84 & 2.06 \\
\hline & L21_(C) & $\mathrm{D}_{2 \mathrm{~h}}$ & 82.02 & 11.98 & 7.28 & 622 & 4.73 & 2.85 & 2.03 \\
\hline
\end{tabular}

A comparison with the previous work, in fig. 4, clearly shows the same relative trend [45-47]. We observed that the calculated electronic gap for bulk germanium is sensitive to the choice of functionals as well as the basis set/pseudopotential. This might explain the difference between the local density approximation (LDA) calculations using non-conserving pseudopotentials of Bruno et al. [45] and the present work. One can clearly see agreement between the LDA calculations using Vanderbilt pseudopotentials of Medaboina et al. [46] and the present work for the smallest-diameter nanowire. However, the divergence for larger diameters can possibly be explained by the difference in nanowire cross-section and/or by the difference between the potentials. On the other hand, our results agree very well with LDA calculations using the exchange and correlation potential of Kholod et al. [47]:

$$
\begin{aligned}
& E(D)_{H L, G e N W_{-} L 10(T)}=(0.66 \pm 0.02)+(15.60 \pm 0.29) \times D^{-1}, \\
& E(D)_{H L, G e N W_{-} L 15(T)}=(0.49 \pm 0.02)+(15.67 \pm 0.27) \times D^{-1}, \\
& E(D)_{H L, G e N W_{-} L 21(T)}=(0.43 \pm 0.02)+(15.56 \pm 0.35) \times D^{-1}
\end{aligned}
$$

The total density of states is shown in fig. 5, for all corresponding diameters of H-GeNWs length L10 (the crosssection of which has been shown in fig. 1). Clearly, in case of tetrahedral (bulk-like, i.e. fig. 1(a)-(e)) H-GeNWs, energy gap is narrowing as far as the diameter of the nanowire is increasing. The comparison of approximately identical diameters of tetrahedral (b) and clathrate (f) shows that the later has a larger gap with a clear red shift. However, we will see in the next section that tetrahedral H-GeNWs are energetically stable compared with clathrate H-GeNWs.

We represent a graphical demonstration of various frontier molecular orbitals in fig. 6 with respect to the crosssection (1.24 nm diameter) and along bulk direction (L10) of hydrogen passivated germanium nanowires selected from fig. 1(b) tetrahedral and (f) clathrate respectively. Along the bulk (length) direction, the HOMO-2, HOMO and $\mathrm{LUMO}+1$ of tetrahedral H-GeNWs is localised everywhere except at both ends of the nanowire. Therefore, the localization of the wave function in LUMO is mainly located at the core of the nanowire which is analogous to the representation of $\mathrm{LUMO}+2$. The HOMO-1, on the other hand exhibits differently, where localisation is distributed on both ends of the nanowire whereas the centre of nanowires is not included. In case of clathrate H-GeNWs, the HOMO-1, HOMO, LUMO and LUMO+2 are localized in the core of the nanowire along the length direction whereas, HOMO-2 and LUMO+1 show random distribution. One can clearly see that the contribution of the wave function to HOMO and LUMO is alternative in tetrahedral H-GeNWs which may indicate the charge transfer and not present in clathrate H-GeNWs. 

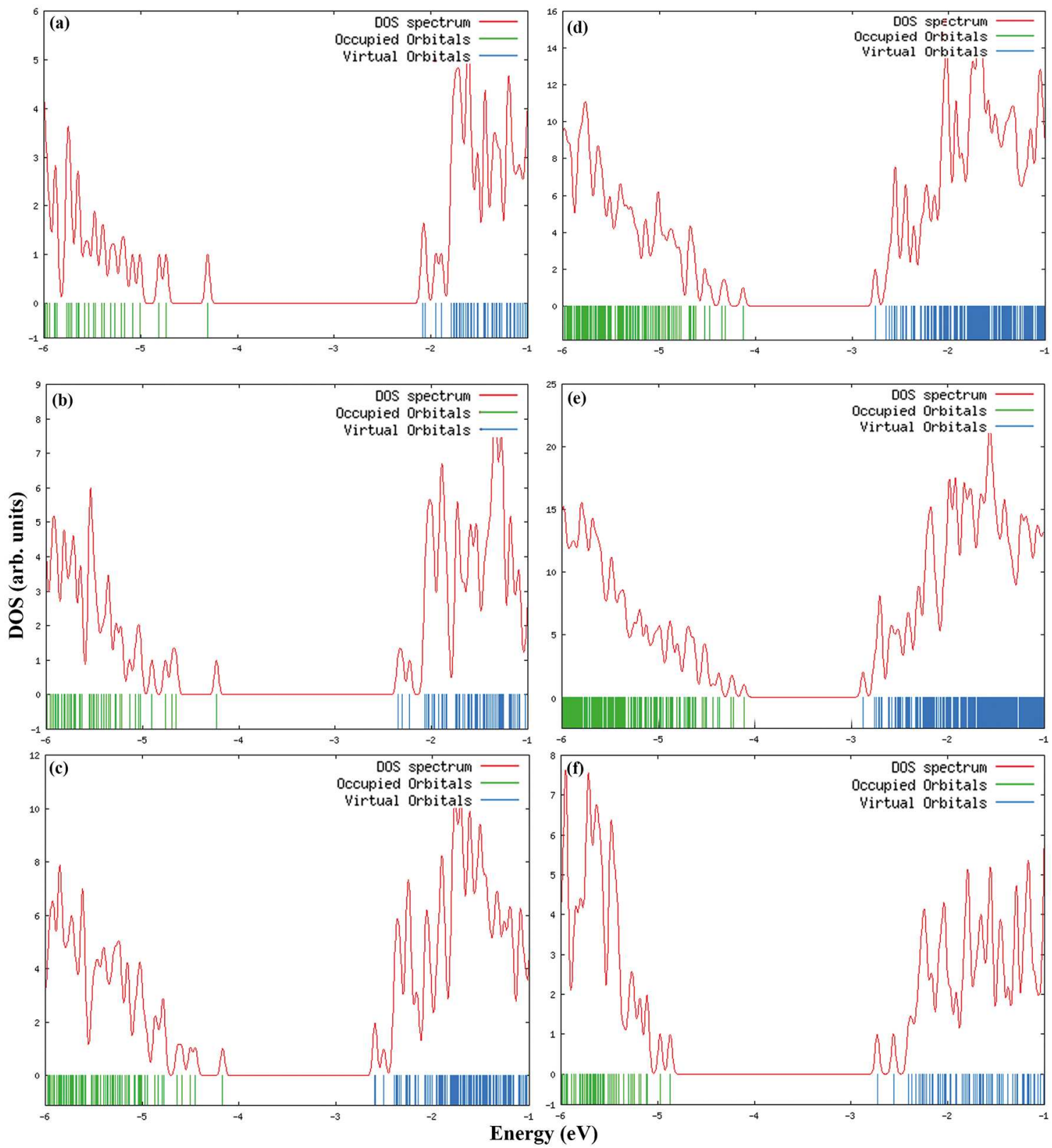

Fig. 5. Representation of density of states (DOS) spectrum for hydrogen passivated germanium nanowires (geometries of which are shown in fig. 1, respectively). The solid red line corresponds to the total DOS, whereas the green and blue vertical lines represent occupied and virtual orbitals respectively. The DFT-PBE@PM6 method is used for calculations.

\subsection{Cohesive properties}

It is well known that the binding energy (per heavy atom) or atomization energy is an absolute comparison with the state where the "molecule" is broken down to its atomic constituents hence can be defined as

$$
B E_{H \ldots G e N W}=N_{G e} E(G e)+N_{H} E(H)-E_{H \ldots G e N W}\left[G e_{N_{G e}} H_{N_{H}}\right]
$$

where $N_{G e}$ and $N_{H}$ represent the total number of germanium and hydrogen atoms respectively within germanium nanowire, $E(G e)$ and $E(H)$ are the energies of single germanium and hydrogen atom respectively and $E_{H \ldots G e N W}$ $\left[G e_{N_{G e}} H_{N_{H}}\right]$ represents the total energy of the nanowire.

Figure 7 shows size dependence of the binding energy per atom with respect to the number of germanium atoms of hydrogen passivated germanium nanowires. It is clear from the figure that the binding energy per atom is decreasing as far as the number of germanium atoms of the nanowire are increasing. As far as the nanowire length (tetrahedral) is increasing from L10 to L21 the binding energy per atom is decreasing. One point from clathrate type nanowires 


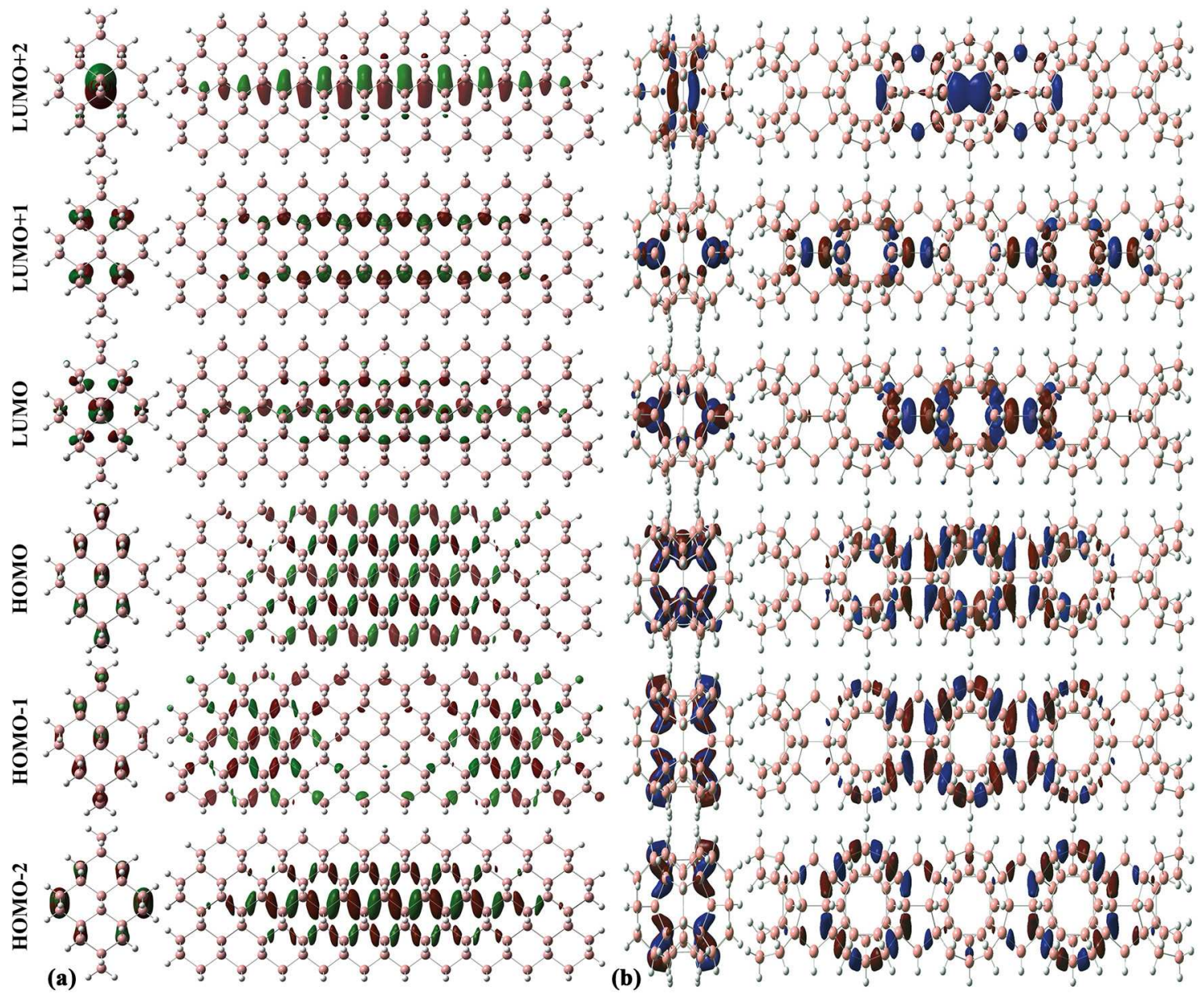

Fig. 6. Graphical representation of various frontier molecular orbitals (FMOs) for hydrogen passivated (a) tetrahedral and (b) clathrate germanium nanowire at isovalue 0.015. The DFT-PBE@PM6 method is used for calculations.

of each representative length is also shown in the figure with lower binding energy values compared with tetrahedral ones:

$$
\begin{aligned}
& E(N)_{B E, H \ldots G e N W_{-} L 10(T)}=(3.04 \pm 0.04)+(10.64 \pm 0.25) \times N^{-\frac{1}{3}}, \\
& E(N)_{B E, H \ldots G e N W_{-} L 15(T)}=(2.96 \pm 0.05)+(12.33 \pm 0.34) \times N^{-\frac{1}{3}} \\
& E(N)_{B E, H \ldots G e N W_{-} L 21(T)}=(2.86 \pm 0.03)+(14.15 \pm 0.26) \times N^{-\frac{1}{3}}
\end{aligned}
$$

Equations (6)-(8) represent the predicted binding energy per germanium atom values using extrapolation fitting the function mentioned in eq. (1). Once again the binding energy per germanium atom is decreasing from $3.04 \mathrm{eV}$ /atom to $2.86 \mathrm{eV}$ /atom as far as length is increasing, however it must be consistent with the experiment value of $3.85 \mathrm{eV} /$ atom [48] for lengths up to infinity. We have already mentioned the reasons in sect. 3.2 .

The cohesive energy deals with the binding energy of the "Ge" core for which the interaction of the surface atoms has been taken into account in a uniform way through the introduction of the chemical potential of hydrogen:

$$
E_{C o h, H \ldots G e N W}=B E_{H \ldots G e N W}+\mu_{H} N_{H},
$$

where $B E_{H \ldots G e N W}$ is the binding energy of the nanowire, $\mu_{H}$ is the chemical potential of hydrogen and $N_{H}$ is the total number of hydrogen atoms in the nanowire:

$$
\begin{aligned}
& E(N)_{C o h, H \ldots G e N W_{-L 10}(T)}=(3.55 \pm 0.02)+(-5.56 \pm 0.12) \times N^{-\frac{1}{3}}, \\
& E(N)_{C o h, H \ldots G e N W_{L} L 15(T)}=(3.59 \pm 0.02)+(-6.46 \pm 0.16) \times N^{-\frac{1}{3}} \\
& E(N)_{C o h, H \ldots G e N W_{-} L 21(T)}=(3.64 \pm 0.02)+(-7.42 \pm 0.15) \times N^{-\frac{1}{3}} .
\end{aligned}
$$




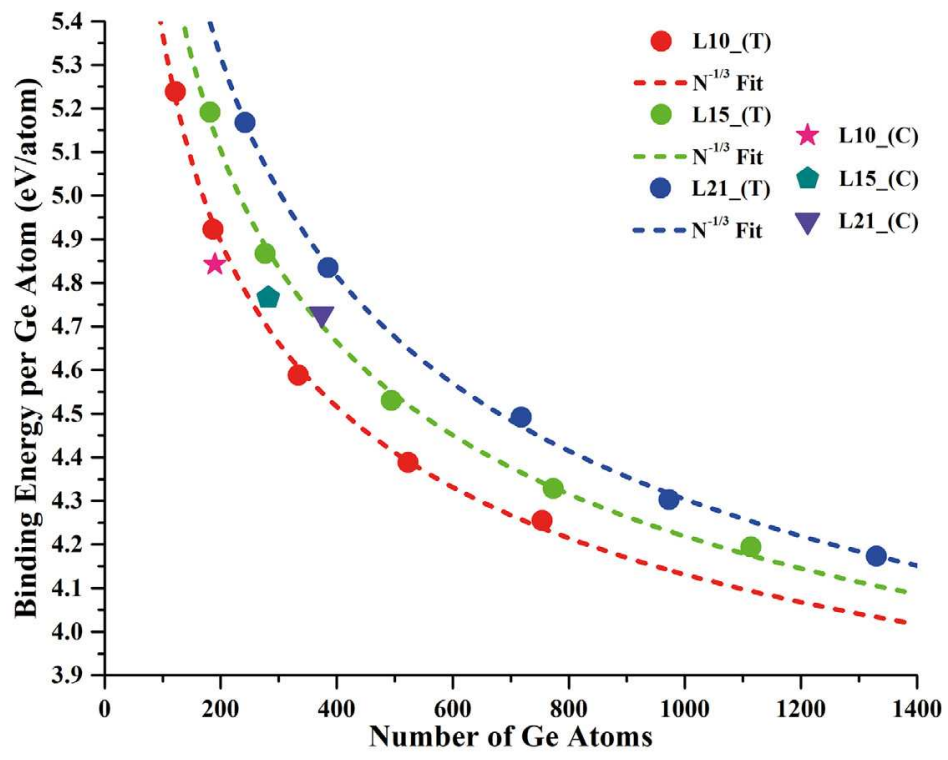

Fig. 7. Binding energy per Ge atom dependence on the size (i.e., number of germanium atoms) of all three different lengths of $\mathrm{H}-$ GeNWs L10 to L21. The red, green and blue dots represent tetrahedral ( $\mathrm{T}$ ) whereas the pink star, cyan pentagon and violet triangle represent clathrate $(\mathrm{C})$ type nanowires. Dotted lines show QC fit using eq. (1). The DFT-PBE@PM6 method is used for calculations.

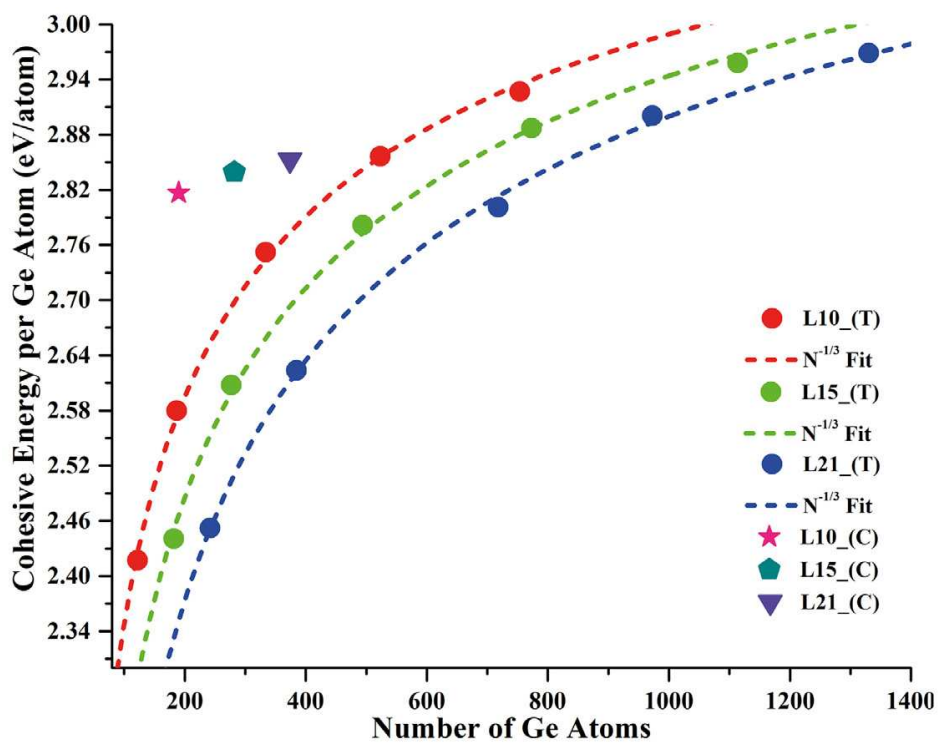

Fig. 8. Cohesive energy per Ge atom dependence on the size (i.e., number of germanium atoms) of all three different lengths of H-GeNWs L10 to L21. The red, green and blue dots represent tetrahedral (T) whereas the pink star, cyan pentagon and violet triangle represent clathrate $(\mathrm{C})$ type nanowires. Dotted lines show QC fit using eq. (1). The DFT-PBE@PM6 method is used for calculations.

Figure 8 shows size dependence of the cohesive energy per atom with respect to the number of germanium atoms of hydrogen passivated germanium nanowires. It is clear from the figure that the cohesive energy per atom is increasing as far as the number of germanium atoms of NW is increasing. As far as the nanowire length (tetrahedral) is increasing from L10 to L21 the cohesive energy per atom is increasing. One point from clathrate type nanowires of each representative length is also shown in the figure with lower cohesive energy values compared with tetrahedral which confirms (in conjunction with binding energy) that clathrate type NWs are less stable compared to tetrahedral ones. 
Surprisingly, a significant improvement in binding energies is observed while introducing the chemical potential to eq. (5). Clearly from eqs. (10)-(12), the cohesive energy is increased from $3.04 \mathrm{eV} /$ atom to $3.64 \mathrm{eV} /$ atom compared with results without the introduction of chemical potential. But it is still far from the experimental agreement, the reasons of which have already been discussed. One can also note that the cohesive energy is improving as far as the size is increasing compared with binding energy (which was decreasing actually). Also, the small cohesive energy difference between the consecutive finite lengths represent that the cohesive energy will eventually be identical for infinite nanowire lengths.

\section{Conclusion}

In summary, we have presented an efficient mixed $a b$ initio and semiempirical method for the cohesive energy and electronic gap calculations of hydrogen passivated germanium nanowires (both tetrahedral and clathrates) with acceptable accuracy, comparable to DFT results, and at a significantly lower computational cost. Taking into account for computational efficiency, in contrast to silicon [49], we have found that for geometry optimization of H-GENWs the PM6 semiempirical method produced the most accurate results compared to the DFT method; whereas other methods such as AM1, PM3 and PM7 clearly underestimate (or overestimate) cohesive/binding energies and gaps compared to the DFT calculations. Therefore PM6, in conjunction with the DFT scheme, i.e., DFT-PBE@PM6, is method of choice for electronic structure calculations of germanium nanowires. However, the bulk energy gap and cohesive energy values out of the QC fitting show underestimated results which is clearly due to the improper selection of functional and basis set/ECP. This is also true when we compared our results with previous data of several other research groups. It is therefore recommended to use more reliable functionals such as hybrid functionals and rather complete basis sets and ECP. Further development in this research work is in progress.

Computational resources from the Turkish Academic Network and Information Center (ULAKBIM) Turkey and the Department of Physics, University of Sargodha (HEC Project \# 172) Pakistan are gratefully acknowledged.

\section{References}

1. Y. Cui, Z. Zhong, D. Wang, W.U. Wang, C.M. Lieber, Nano Lett. 3, 149 (2003).

2. Y. Huang, X. Duan, Y. Cui, L.J. Lauhon, K. Kim, C.M. Lieber, Science 294, 1313 (2001).

3. Y. Cui, Q. Wei, H. Park, C.M. Lieber, Science 293, 1289 (2001).

4. B. Polyakov, B. Daly, J. Prikulis, V. Lisauskas, B. Vengalis, M.A. Morris, J.D. Holmes, D. Erts, Adv. Mater. 18, 1812 (2006).

5. Y. Wu, P.D. Yang, Chem. Mater. 12, 605 (2000).

6. B. Yu, X.H. Sun, G.A. Calebotta, G.R. Dholakia, M. Meyyappan, J. Clust. Sci. 17, 579 (2006).

7. S. Patibandla, S. Pramanik, S. Bandyopadhyaya, G.C. Tepper, J. Appl. Phys. 100, 044303 (2006).

8. E. Garnett, P. Yang, Nano Lett. 10, 1082 (2010).

9. C.K. Chan, X.F. Zhang, Y. Cui, Nano Lett. 8, 307 (2008).

10. Y.H. Ahn, J. Park, Appl. Phys. Lett. 91, 162102 (2007).

11. M.C. McAlpine, H. Ahmad, D. Wang, J.R. Heath, Nat. Mater. 6, 379 (2007).

12. M. Amato, S. Ossicini, R. Rurali, Nano Lett. 11, 594 (2011).

13. D. Wang, Y. Chang, Z. Liu, H. Dai, J. Am. Chem. Soc. 127, 11871 (2005).

14. P. Nguyen, H.T. Ng, M. Meyyappan, Adv. Mater. 17, 549 (2005).

15. D. Wang, Q. Wang, A. Javey, R. Tu, H.J. Dai, H. Kim, P.C. McIntyre, T. Krishnamohan, K.C. Saraswat, Appl. Phys. Lett. 83, 2432 (2003).

16. A.B. Greytak, L.J. Lauhon, M.S. Gudiksen, C.M. Lieber, Appl. Phys. Lett. 84, 4176 (2004).

17. S. Niaz, S. Slimani, M.A. Badar, G. Subhan, M.A. Khan, Sensors Transducers 189, 162 (2015).

18. A. Nduwimana, X.Q. Wang, J. Phys. Chem. C 114, 9702 (2010).

19. H. Adhikari, P.C. McIntyre, S.Y. Sun, P. Pianetta, C.E.D. Chidsey, Appl. Phys. Lett. 87, 263109 (2005).

20. G. Collins, P. Fleming, S. Barth, C. O'Dwyer, J.J. Boland, M.A. Morris, J.D. Holmes, Chem. Mater. 22, 6370 (2010).

21. D.W. Wang, Y.L. Chang, Z. Liu, H.J. Dai, J. Am. Chem. Soc. 127, 11871 (2005).

22. J.T. Arantes, A. Fazzio, Nanotechnology 18, 295706 (2007).

23. S.P. Beckman, J. Han, J.R. Chelikowsky, Phys. Rev. B 74, 165314 (2006).

24. M. Bescond, N. Cavassilas, K. Nehari, M. Lannoo, J. Comput. Electron. 6, 341 (2007).

25. C. Harris, E.P. O'Reilly, Physica E 32, 341 (2006).

26. D. Medaboina, V. Gade, S.K.R. Patil, S.V. Khare, Phys. Rev. B 76, 205327 (2007).

27. M. Jing, M. Ni, W. Song, J. Lu, Z. Gao, L. Lai, W.N. Mei, D. Yu, H. Ye, L. Wang, J. Phys. Chem. B 110, 18332 (2006).

28. J.P. Perdew, K. Burke, M. Ernzerhof, Phys. Rev. Lett. 77, 3865 (1996).

29. G. Igel-Mann, H. Stoll, H. Preuss, Mol. Phys. 65, 1321 (1988).

30. A. Bergner, M. Dolg, W. Kuechle, H. Stoll, H. Preuss, Mol. Phys. 80, 1431 (1993). 
31. M.J. Frisch, G.W. Trucks, H.B. Schlegel, G.E. Scuseria, M.A. Robb, J.R. Cheeseman, G. Scalmani, V. Barone, B. Mennucci, G.A. Petersson, H. Nakatsuji, (1990). Gaussian 09 (Gaussian. Inc., Wallingford, CT, USA, 2009) 542.

32. M.J.S. Dewar, E.G. Zoebisch, E.F. Healy, J.J.P. Stewart, J. Am. Chem. Soc. 107, 3902 (1985).

33. M.J.S. Dewar, J. Caoxian, Organometallics 8, 1544 (1989).

34. M.J.S. Dewar, J. Comput. Chem. 10, 221 (1989).

35. M.J.S. Dewar, J. Mol. Modeling 13, 1173 (2007).

36. J.J.P. Stewart, J. Mol. Modeling 19, 1 (2013).

37. MOPAC2012, J.J.P. Stewart, Stewart Computational Chemistry, http://OpenMOPAC.net (Colorado Springs, CO, USA, 2012).

38. MOPAC2016, J.J.P. Stewart, Stewart Computational Chemistry, http://OpenMOPAC.net (Colorado Springs, CO, USA, 2016).

39. L. Tian, F. Chen, J. Comput. Chem. 33, 580 (2012).

40. N.M. O'Boyle, A.L. Tenderholt, K.M. Langner, J. Comput. Chem. 29, 839 (2008).

41. S. Niaz, A.D. Zdetsis, J. Phys. Chem. C 120, 11288 (2016).

42. S. Niaz, E.N. Koukaras, N.P. Katsougrakis, T.G. Kourelis, D.K. Kougias, A.D. Zdetsis, Microelectron. Eng. 112, 231 (2013).

43. C. Kittel, Introduction to Solid State Physics, 6th ed. (John Wiley \& Sons, New York, 1986).

44. S. Niaz, A.D. Zdetsis, M.A. Badar, S. Hussain, I. Sadiq, M.A. Khan, J. Chem. Soc. Pak. 38, 207 (2016).

45. M. Bruno, M. Palummo, S. Ossicini, R. Del Sole, Surf. Sci. 601, 2707 (2007).

46. D. Medaboina, V. Gade, S.K.R. Patil, S.V. Khare, Phys. Rev. B 76, 205327 (2007).

47. A.N. Kholod, V.L. Shaposhnikov, N. Sobolev, V.E. Borisenko, F.A. D'Avitaya, S. Ossicini, Phys. Rev. B 70, 035317 (2004).

48. C. Kittel, Introduction to Solid State Physics, 8th ed. (John Wiley \& Sons, Hoboken, NJ, 2005).

49. E.N. Koukaras, S. Niaz, D.A. Zdetsis, A.D. Zdetsis, Microelectron. Eng. 90, 88 (2012). 